\title{
The illuminance of laryngoscopes at two central hospitals
}

\section{GA Davies, H Perrie, J Scribante, PC Anamourlis}

Department of Anaesthesiology, School of Clinical Medicine, Faculty of Health Sciences, University of the Witwatersrand, Johannesburg, South Africa

Corresponding author, email: gwynethad@gmail.com

Background: Direct laryngoscopy and successful endotracheal intubation require optimal illumination of laryngeal structures. The International Organization of Standardization (ISO) describes minimum adequate laryngoscope illuminance as 500 lux after 10 minutes, and further describes optimal dimensions of the illumination field. Laryngoscope light is subjectively assessed by the anaesthetist as part of theatre preparation. This study sought to describe the illumination of laryngoscopes at two academic hospitals, to compare illumination of incandescent and fibreoptic laryngoscopes and to compare the accuracy of a mobile phone application (app) to a lux meter.

Methods: A prospective, contextual, descriptive study was conducted, testing the illumination of 43 laryngoscopes with a lux meter, as well as a mobile phone app. The illumination field size of each laryngoscope was determined.

Results: ISO Standard for illumination was met by 8 (18.6\%) laryngoscopes, and 11 (25.5\%) had an adequate illumination field. Only 4 (9.3\%) laryngoscopes met both criteria. The mobile phone app readings were significantly different from those obtained with a lux meter $(p=0.0008$ ). After battery replacement 23 further laryngoscopes demonstrated an adequate illuminance. No significant difference was found between incandescent and fibreoptic laryngoscope illuminance $(p=0.86)$.

Conclusion: This study demonstrated that the available laryngoscopes had poor illuminance. A mobile phone app was not comparable to a lux meter. Routine objective illuminance testing as well as regular battery changes are suggested to be implemented.

Keywords: laryngoscope, direct laryngoscopy, tracheal intubation, illumination, mobile phone app

\section{Introduction}

Direct laryngoscopy allows for inspection of airway structures, removal of foreign bodies, and intubation of the airway. Despite the availability of sophisticated equipment such as video laryngoscopes and flexible fibreoptics, the rigid laryngoscope remains the gold standard for direct laryngoscopy due to its simple design and ease of use.

A laryngoscope is required to produce adequate illuminance of the airway structures. A functioning laryngoscope is listed in the South African Society of Anaesthesiologists (SASA) Airway Guidelines ${ }^{1}$ as essential as part of the minimum safety requirements. The SASA Practice Guidelines of $2013^{2}$ stipulate that the anaesthetist should check all such theatre equipment. Studies previously conducted have shown that the light produced may be of varying intensity and that the laryngoscopes functionality may not always be reliable. ${ }^{3-8}$

The two most common lighting mechanisms of laryngoscopes are the "bulb-on-blade" or incandescent type, and the "bulbin-handle" or fibreoptic type. The light source or bulb of an incandescent laryngoscope is mounted on the blade of the laryngoscope. In contrast, the light source of a fibreoptic laryngoscope is encased within the handle, and is conducted to the distal portion of the blade by a glass or plastic fibre bundle. ${ }^{9}$ Studies have demonstrated the superior illuminance of the fibreoptic laryngoscopes when compared to the incandescent type. ${ }^{4,7,8}$
Illuminance is the amount of light falling on a particular object, measured in lumens per square meter, or lux. Factors have been identified which influence the illuminance of laryngoscope light, and include the lighting mechanism (incandescent or fibreoptic), wear and tear of components due to repetitive cleaning and the battery life as well as type of battery used. ${ }^{9}$ In addition, a study by Levitan et al. ${ }^{5}$ showed that, with regards to incandescent laryngoscopes, the distance between the bulb and the tip of the laryngoscope blade will affect the illuminance of the airway structures. Macintosh, the inventor of the curved blade, did not stipulate the actual angle or dimensions of the blade, as he felt they were not of primary importance in direct laryngoscopy. ${ }^{10}$ Hence, although named "Macintosh" laryngoscope blades, the size of the flange, blade curve and bulb placement vary according to manufacturer, and are usually titled by country of origin, namely "American, English or German". Levitan et al. ${ }^{5}$ noted the superiority of the shorter distance between bulb and blade tip of the German blade in comparison to a longer distance of the American blades.

In the clinical setting the illuminance of a laryngoscope is subjectively assessed by the user. The International Standard Organisation (ISO) Standard 7376:200911 requires a more sophisticated measurement using a lux meter. The Standard specifies a minimum illuminance of 500 lux for at least 10 minutes. In comparison, the ambient indoor lighting is between 150 to 500 lux, and light in operating theatres is typically about 1000 lux. ${ }^{12}$ The Standard further describes the required dimensions of the field of light distributed by the laryngoscope. The ISO Standard 7376:2009 is in the process of 


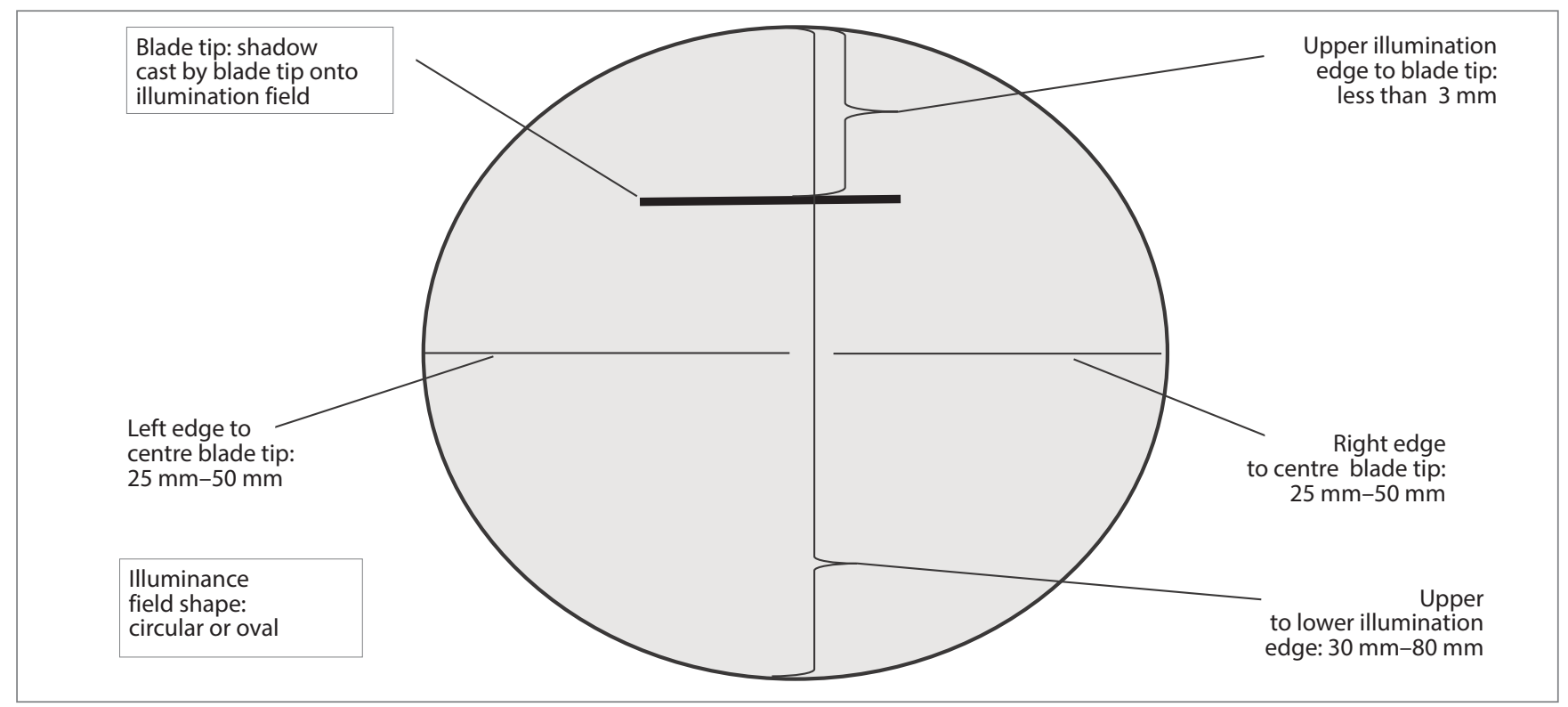

Figure 1: Graphic representation of illumination field and required dimensions

being updated, with more detailed instructions on demarcating the illuminance field described in the draft version. ${ }^{13} \mathrm{~A}$ study by Murphy et al. ${ }^{7}$ of 691 laryngoscopes found that $28 \%$ did not meet the minimum required 500 lux at 10 minutes. Baker et al. ${ }^{14}$ found in their study that only $11 \%$ of laryngoscope illuminance audited exceeded 500 lux, and none complied with the dimensions of the illumination field.

With advances in technology and mobile phone applications (apps) the users may have access to sophisticated equipment in their pockets. There are mobile phone lux meter apps available that make use of the mobile phone camera lens as the light sensor. However, not all are accredited. The company DIAL, which produces lux meter devices, conducted a study of various free lux meter apps available in comparison to one of their standard lux meter devices. Their findings showed that the lux meter app readings were variable, and were not consistent with those taken by the lux meter. ${ }^{15}$ In contrast, a study by Machado et al. ${ }^{6}$ measuring illuminance of laryngoscopes using an app and a lux meter showed that the app was as accurate as a lux meter. The authors describe the type of phone used in the study (Motorola ${ }^{\circledR}$ Moto G XT1068), but not the android app chosen, or if any other accessories were required.

No South African studies were identified that described the illuminance of laryngoscope light. A study was undertaken with the aim to describe the illuminance of the laryngoscopes at two hospitals affiliated to the Department of Anaesthesiology at the University of the Witwatersrand (Wits), Charlotte Maxeke Johannesburg Academic Hospital and Chris Hani Baragwanath Academic Hospital. This study sought to describe the illumination of laryngoscopes at two academic hospitals, to compare illumination of incandescent and fibreoptic laryngoscopes and to compare the accuracy of a mobile phone application (app) to a lux meter.

\section{Methodology}

The research design was prospective, contextual and descriptive. All 43 theatres in use were included in the study. A total of 43 laryngoscopes and size 3 blades were tested. A convenience sampling method was used. The size 3 blade was chosen as it is most frequently used. The Dr.Meter Digital Illuminance/ Light Meter LX1330B was used. The manufacturer specifies an accuracy of within $2 \%$. This meter was chosen because it was the most cost effective. An iPhone 7 was used. The app, Lux Camera 2.0, was selected as it was simple to use, free to download from the South African app store and did not require the purchase of extra accessories. It received a "fair' rating on AppCrawlr.com, ${ }^{16}$ and was the only free app that had been rated. A portable dark

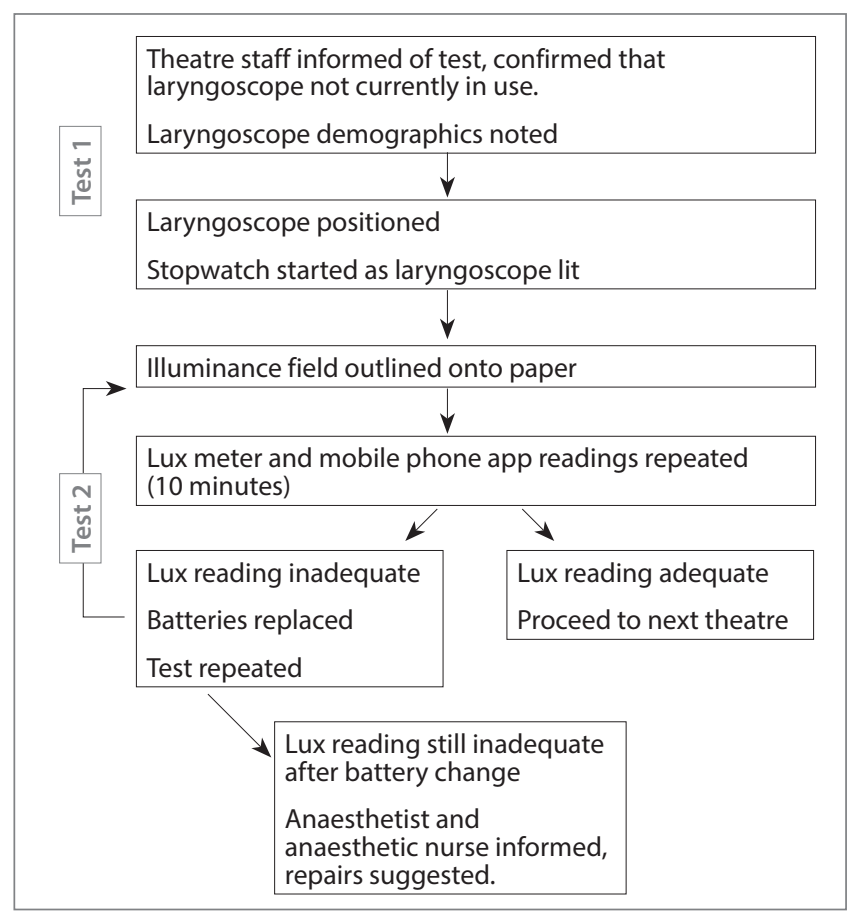

Figure 2: Data collection process 
box was constructed similarly to those in previous studies ${ }^{4,8}$ and was used to minimise ambient light.

The illumination field was traced onto paper and measured as shown in Figure 1 in accordance with the ISO 7376:2009 Standard. ${ }^{11}$ The illumination field was either adequate (fulfilled all criteria) or inadequate.

The ISO 7376:2009 Standard ${ }^{11}$ requires two sets of illuminance measurements to be taken, the first as the laryngoscope is initially assembled and the light is initially switched on, and the second after the light has been lit for 10 minutes. The Standard is met if an illuminance is initially of over 500 lux and is maintained above that level for at least 10 minutes.

The first set of tests with the lux meter and mobile phone app has been referred to as "Test 1". A second set of data was collected after the batteries of laryngoscopes, with illuminance deemed to be inadequate, were replaced with new batteries. This set of data is referred to as "Test 2". All replacement batteries were new Duracell ${ }^{\circledR}$ alkaline $1.5 \mathrm{~V}$ batteries, either size AA or $\mathrm{C}$ (depending on the handle size). All old batteries were disposed of responsibly. Figure 2 shows the process of how the data were collected.

The data were entered onto a Microsoft Excel spreadsheet. Descriptive and inferential statistics using STATA (StataCorp, USA) and Graphpad Prism 7 (GraphPad Software, Inc, USA) were used for analysis. A Wilcoxon signed-rank test was used to compare data, with a $p<0.05$ considered statistically significant.

\section{Results}

All 43 laryngoscopes were of the reusable type. Twenty-eight $(65.1 \%)$ of the laryngoscope handle and blade pairs were mismatched sets not from the same manufacturer, which could possibly contribute to poor electrical contact between the parts and affect illumination. Thirteen (30.2\%) laryngoscopes were incandescent and 30 (69.7\%) were fibreoptic.

The number of handles and blades made by various manufacturers are shown in Table I. The majority were made by Welch Allyn ${ }^{\circledast}$, those labelled as "other" included Intubrite ${ }^{\circledR}$, Sololite ${ }^{\circledR}$, Vibgyor ${ }^{\circledast}$, Rheister, Ultra ${ }^{\circledR}$, Penlon ${ }^{\circledast}$, Hi-care ${ }^{\oplus}$, Gale Med ${ }^{\circledR}$, Mdlunalite ${ }^{\circledast}$, Rhein, $\mathrm{EFF}^{\oplus}$, and Medicon. One blade, manufactured by Intubrite, had 2 light bulbs; 1 light emitting diode type (LED) and 1 ultraviolet (UV) bulb.

Fourteen (32.5\%) handles were of the paediatric type (using AA batteries), while the remaining $67 \%$ were the standard size (housing size $C$ batteries). The batteries were all alkaline and included 27 sets of Eveready ${ }^{\circledR}, 9$ Energizer ${ }^{\circledR}, 5$ Excell ${ }^{\circledR}, 1$ Duracell ${ }^{\circledR}$, and 1 Super Power ${ }^{\circledast}$.
Only 11 (25.6\%) laryngoscopes had initially acceptable lux readings at 0 minutes, of these $3(6.9 \%)$ were considered inadequate as their readings of above 500 lux were not maintained for 10 minutes. Eight (18.6\%) laryngoscopes had an illuminance of over 500 lux after 10 minutes. Three (6.9\%) laryngoscopes had adequate illuminance at 0 and 10 minutes when tested with the app. The findings of Test 1 and Test 2 (with new batteries) are shown in Figure 3. The results of testing with the lux meter at 10 minutes are highlighted in Figure 3 as these results comply with the ISO 7376:2009 Standard with regards to illumination after 10 minutes tested with a lux meter.

Six of the 8 laryngoscopes that met the ISO Standard in Test 1 were matching Welch Allyn handle and blade pairs. Out of the 12 laryngoscopes that did not meet the ISO Standard after a battery change in Test 2, 10 were mismatched handle and blade pairs.

The differences between the illuminance readings on the lux meter compared to the mobile app were statistically significant at 0 minutes $(p=0.001)$ and 10 minutes $(p=0.008)$.

Of the 8 laryngoscopes that met the ISO Standard after 10 minutes, 1 was incandescent and 7 were fibreoptic. The difference between the illuminance of the 2 types of laryngoscopes was found not to be statistically significant $(p=0.86)$.

Eleven (25.6\%) laryngoscopes had an adequate illuminance field in Test 1 . Only 1 (2.3\%) laryngoscope had readings of over 500

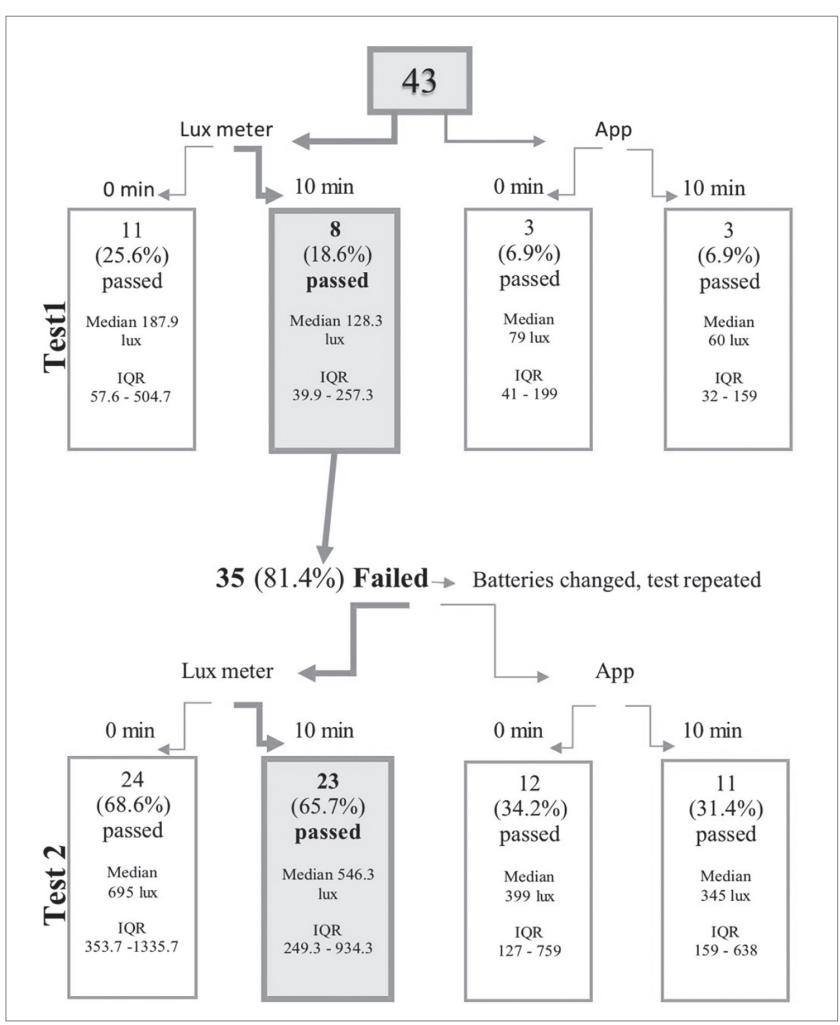

Figure 3: Results of Test 1 and Test 2

Table I: Manufacturers of blades and handles

\begin{tabular}{|c|c|c|c|c|c|c|}
\hline \multirow[b]{2}{*}{ Manufacturer } & \multicolumn{6}{|c|}{ Number (percentage) } \\
\hline & Welch Allyn & Heine & Medisensor & No name & Hilbro & Other \\
\hline Handle & $25(58)$ & $0(0)$ & $3(6.9)$ & $4(9.3)$ & $3(6.9)$ & $8(18.6)$ \\
\hline Blade & $23(53)$ & $4(9.3)$ & $1(2.3)$ & $5(11.6)$ & $0(0)$ & $10(23)$ \\
\hline
\end{tabular}


lux for both the mobile phone app and the lux meter, as well as having an adequate illuminance field. A further 15 (34.9\%) laryngoscopes' illumination fields became adequate after the batteries were changed in Test 2 .

\section{Discussion}

The findings of this study were in keeping with previous studies, $5,7,14$ as well as the clinical impression of poor laryngoscope illuminance. Only 8 (18.6\%) laryngoscopes of the 43 tested met the ISO Standard after 10 minutes. Compared to theatre ambient and surgical light requirements, a minimum of 500 lux is seemingly low. ${ }^{12}$

The importance of noting the manufacturer of the laryngoscope handles and blades has been highlighted in previous studies. ${ }^{5}$ Although the length of the Macintosh size 3 blade is standardised, the degree of the curve, the size of the flange, and the placement of the light bulb/fibreoptic bundle do vary. The latter is suggested to have an effect on the illuminance. The majority of handles (58\%) and blades (53\%) in use in the two hospitals were made by Welch Allyn, an American manufacturer. It would be difficult to assess which manufacturer had the best illuminance, considering that there were many single handle and blade sets from different manufacturers. Mismatched pairs of laryngoscope handles and blades may lead to poor electrical contact and affect illumination. It is not known whether the Welch Allyn laryngoscopes were purchased more recently, and therefore had not yet been exposed to heavy use and cleaning.

All laryngoscopes tested were reusable. The methods of cleaning and autoclaving of reusable handles and blades has been previously shown to cause wear and tear and ultimately affect their functioning. ${ }^{17,18}$ This study did not take into consideration how the equipment was being cleaned, and it is not known how long each handle and blade had been in use. It may be considered that the laryngoscopes that did not meet criteria after Test 2 were old. Faulty electrical connections, damaged fibre bundles of the fibreoptic blades and exhausted light bulbs are all possible causes of low illuminance, ${ }^{4}$ which could be the topic of a future study.

The Intubrite laryngoscope blade had two bulbs, one LED and one UV. This laryngoscope passed the first test with an average illuminance of 827 lux at 10 minutes. This illuminance was not the highest of the study group, as one would assume. This may be because it was paired with a handle from a different company (Sololite), or perhaps the batteries may have been partially depleted. No second test with new batteries was performed on this particular laryngoscope, as it met the ISO Standard in Test 1. The manufacturer of this particular blade specifies that the quality of light produced allows for better tissue differentiation and less glare, but not necessarily increased illumination. ${ }^{19}$

The reason for the required 10-minute time duration for maintaining an illuminance of 500 lux is not specified in the ISO Standard. It may be to ensure the adequacy of the illuminance in a situation such as a difficult airway where the laryngoscope may be left on for a longer duration. In Test 1, although 11 (25.6\%) laryngoscopes had adequate illuminance at 0 minutes, 3 (6.9\%) did not maintain adequate illuminance. A possible reason is that the batteries were old and depleted. Test 2 showed that 23 laryngoscopes (65.7\% of the repeated 35 tests) met the ISO Standard after new batteries were inserted. A weakness of this study is that the types of batteries were noted but were not tested with a multimeter to determine their condition.

There was no significant difference found between the illuminance of the incandescent or fibreoptic laryngoscopes $(p=$ 0.86 ), which is in keeping with a study by Volsky et al. ${ }^{8}$ In contrast, Murphy et al. ${ }^{7}$ found the fibreoptic illuminance to be superior to the incandescent. None of the laryngoscopes in this study were made with bulbs utilising xenon, which the authors of the studies mentioned had found to be superior to incandescent and fibreoptic. A Canadian study ${ }^{20}$ found the incandescent light source was superior to the fibreoptic. In their study, the ISO Standard was not used to determine illuminance. The authors measured the exposure indices of the laryngoscopes and converted their readings into lux by a complex calculation.

The mobile phone app was not found to be accurate when compared to a lux meter. This is in keeping with a previous comparison of various free apps to a lux meter, ${ }^{15}$ but is in contrast to the study by Machado et al. ${ }^{6}$ The author specified that the app used would have an accuracy of $80 \%$ in the range of $5-10000$ lux, but it is not mentioned which app was used, if the app was purchased, and if any other accessories were required to ensure accuracy.

Only $25.6 \%$ of laryngoscopes had an adequate illuminance field. This may pose a problem in the case of a difficult airway, where the glottic opening is not directly in front of the laryngoscope blade tip. Potential damage to soft tissue and teeth could occur if the anaesthetist were to repeatedly reposition a blade to bring the glottis into a narrow illuminance field. A further $34.9 \%$ of laryngoscopes demonstrated adequate illuminance fields after the batteries were replaced, indicating the importance of using non-depleted batteries.

During this study, if a laryngoscope was still found to have inadequate illuminance after a battery change, the findings were reported to the anaesthetist and anaesthetic nurse assigned to the particular theatre. They were then responsible for reporting faulty equipment and sending the laryngoscope for repairs. The particular theatre could continue operating at the discretion of the theatre staff. This may pose an ethical issue, as the staff may have continued to use the laryngoscope on patients despite the proven inadequacy of their equipment. There are, however, no studies proving the relationship between adequate laryngoscope lighting and successful intubation. It may have been of value to have followed up the faulty laryngoscopes sent for repairs, to determine the cause of their low illuminance, and if they could be returned to circulation after repairs were carried out. 
Of the laryngoscopes that were in use, $81.4 \%$ had inadequate illuminance, and $74.4 \%$ had an inadequate illuminance field. The improvements seen between Test 1 and Test 2 suggest that more regular battery changes may improve illuminance. The increased cost of the batteries is outweighed by the potential improvement in conditions for direct laryngoscopy. Regular audits of laryngoscope illuminance using a lux meter may be of benefit in providing objective data that may be used as motivation to replace older equipment. Furthermore, a cleaning and repair register may be of value. An inexpensive, easy to use, mobile phone app that is comparable to a lux meter was not found by this study.

\section{Conclusion}

The anaesthetic complications of failed airway management result in significant morbidity as well as mortality. An anaesthetist should therefore have optimal tools available for successful intubation, such as a laryngoscope with optimal illumination. This study concludes that regular objective audits of laryngoscope illuminance, frequent battery changing and documentation of laryngoscope cleaning and sterilisation may assist in improving the illuminance if the laryngoscopes in use at $\mathrm{CHBAH}$ and $\mathrm{CMJAH}$. A further consideration may be to attempt to have matching handle and blade sets from a single manufacturer available in theatre.

\section{Ethics approval}

Approval to conduct the study was obtained from the Wits Human Research Ethics Committee (Medical) and other relevant authorities.

\section{Conflict of interest}

We declare that we have no financial or personal relationships which may have inappropriately influenced us in writing this paper.

\section{Acknowledgements}

This research was done in partial fulfilment of a Master of a Medicine degree.

\section{References}

1. Hodgson E, Milner A, Alberts A, Roos J, Reyneke M. SASA Airway Guidelines. SAJAA. 2014;20(4):3-4

2. Bettings $P$, Diedericks J, Fourie $P$, Joubert I, Kluyts $H$, Lundgren $C$, et al. SASA Practice Guidelines 2013. SAJAA. 2012;19(1):5.

3. Cheung K, Kovacs G, Law J. Illumination of bulb-on-blade laryngoscopes in the out-of-hospital setting. Acad Emerg Med. 2007;14:496-9.

4. Howes B. The reliability of laryngoscope lights. Anaesthesia. 2006;61(5):488-91. doi: http://dx.doi.org/10.1111/j.1365-2044.2006.04611.x.

5. Levitan R, Kelly J, Kinkle W, Fasano C. Light intensity of curved laryngoscope blades in Philadelphia Emergency Departments. Ann of Emerg Med. 2007;50(3):253-7. doi: https://doi.org/10.1016/j.annemergmed.2007.05.005.

6. Machado D, Esteves D, Branca P. Accuracy of a smartphone to test laryngoscope's light and an audit of our laryngoscopes using an ISO standard. Rev Bras Anestesiol. 2016;32(2):180-3. doi: http://dx.doi.org/10.1016/j.bjane.2016.07.007.

7. Murphy M, Volsky $P$, Darrow D. Laryngoscope illuminance in a tertiary care medical center: industry standards and implications for quality laryngoscopy. JAMA Otolaryngol Head Neck Surg. 2015;153(5):806-11. doi: http://dx.doi. org/10.1177/0194599815587700.

8. Volsky P, Murphy M, Darrow D. Laryngoscope illuminance in a tertiary children's hospital: Implications for quality laryngoscopy. JAMA Otolaryngol Head Neck Surg. 2014;140(7):603-7. doi: http://dx.doi.org/10.1001/jamaoto.2014.676.

9. Levitan R, Kovacs G. Direct Laryngoscopy. In: Hung O, Murphy M, editors. Hung's Difficult and Failed Airway Management, 3e. New York, NY: McGraw-Hill Education; 2017.

10. Macintosh R. A new laryngoscope. Lancet. 1943;241(6233):205. doi: https://doi. org/10.1016/S0140-6736(00)89390-3.

11. The International Organization for Standardization. 7376:2009. Anaesthetic and respiratory equipment- Laryngoscopes for tracheal intubation. 2 ed. Switzerland: ISO; 2009. p. 1-34

12. EngineeringToolBox. Illuminance- Recommended Light Level [Internet] 2004 [01/12/2015]. Available from: https://www.engineeringtoolbox.com/light-levelrooms-d_708.html. [Accessed 1 Nov 2018].

13. The International Organization for Standardization. DIS7376. Anaesthetic and Respiratory Equipment- Laryngoscopes for tracheal intubation; 2018. p. 15-6.

14. Baker P, McQuoid S, Thompson J, Jacobs R. An audit of laryngoscopes and application of a new ISO standard. Pediatric Anesthesia. 2011;21(4):428-34.

15. DIAL. Luxmeter app versus measuring device: are smartphones suitable for measuring illuminance? [Internet]. Lüdenscheid, Germany: DIAL gmbH. 2016. Available from: https://www.dial.de/en/blog/article/luxmeter-app-versusmeasuring-deviceare-smartphones-suitable-for- measuring-illuminance/ [Accessed 2 Dec 2016].

16. AppCrawlr. Best iOS apps for light meters [Internet]: Softonic international S.A. 2015. Available from: http://www.appcrawlr.com/app/search?q=light+meter\&de vice=ios\&price=Free. [Accessed 31 Nov 2016].

17. Bucx M, De Gast H, Veldhuis J. The effect of mechanical cleaning and thermal disinfection on light intensity provided by fibrelight Macintosh laryngoscopes. Anaesthesia. 2003;58:461-5. doi: https://doi. org/10.1046/j.1365-2044.2003.03124.x

18. Bucx M, Veldman D, Beenhakker $M$. The effect of steam sterilization at 134 degrees $C$ on light intensity provided by fibrelight Macintosh laryngoscopes. Anaesthesia. 1999;54:875-8. doi: https://doi. org/10.1046/j.1365-2044.1999.00948.x.

19. Intubrite. Laryngoscope Blades California USA: IntuBrite; [31/11/2015]. Available from: https://intubrite.com/products/laryngoscope-blades/ [Accessed 1 Nov 2018].

20. Tousignant G, Tessler M. Light intensity and area of illumination provided by various laryngoscope blades. Can J Anaesth. 1994;41(865). 